International Journal of Pure and Applied Mathematics

Volume 98 No. 4 2015, 443-455

ISSN: 1311-8080 (printed version); ISSN: 1314-3395 (on-line version)

url: http://www.ijpam.eu

doi: http://dx.doi.org/10.12732/ijpam.v98i4.3

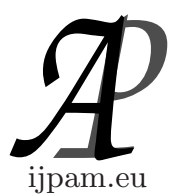

\title{
PSEUDO t-NORMS AND FUZZY CONNECTIVES
}

\author{
Yong Chan Kim \\ Department of Mathematics \\ Gangneung-Wonju University \\ Gangneung, Gangwondo 210-702, KOREA
}

\begin{abstract}
In this paper, we investigated the properties of pseudo t-conorms and implications induced by pairs of negations and a pseudo t-norm. Moreover, we give their examples.
\end{abstract}

AMS Subject Classification: 03E72, 03G10, 06A15, 06F07

Key Words: pseudo t-norms, pseudo t-conorms, pairs of negations, pairs of implications

\section{Introduction}

Many researchers [1-8] introduced non-commutative algebraic structures. This concept is motivated not only by the logically interest, but also by the relation with some remarkable mathematical structures. In [3], pseudo t-norms were investigated in bounded lattices in a sense as non-commutative property. Kim [10] introduced pairs of negations and implications. which are induced by noncommutative property.

In this paper, we investigated the properties of pseudo t-conorms and implications induced by pairs of negations and a pseudo t-norm. Moreover, we give their examples.

Received: $\quad$ May 17, 2014

(c) 2015 Academic Publications, Ltd. url: www.acadpubl.eu 


\section{Preliminaries}

In this paper, we assume that $(L, \vee, \wedge, \perp, \top)$ is a bounded lattice with a bottom element $\perp$ and a top element $T$.

Definition 2.1.[3,6] A map $T: L \times L \rightarrow L$ is called a pseudo t-norm if it satisfies the following conditions:

(T1) $T(x, T(y, z))=T(T(x, y), z)$ for all $x, y, z \in L$,

(T2) If $y \leq z, T(x, y) \leq T(x, z)$ and $T(y, x) \leq T(z, x)$,

(T3) $T(x, \top)=T(\top, x)=x$.

A pseudo t-norm is called a $t$-norm if $T(x, y)=T(y, x)$ for $x, y \in L$

A map $S: L \times L \rightarrow L$ is called a pseudo t-conorm if it satisfies the following conditions:

(S1) $S(x, S(y, z))=S(S(x, y), z)$ for all $x, y, z \in L$,

(S2) If $y \leq z, S(x, y) \leq S(x, z)$ and $S(y, x) \leq S(z, x)$,

(S3) $S(x, \perp)=S(\perp, x)=x$.

A pseudo t-conorm is called a $t$-conorm if $S(x, y)=S(y, x)$ for $x, y \in L$.

Definition 2.2.[10] A pair $\left(n_{1}, n_{2}\right)$ with maps $n_{i}: L \rightarrow L$ is called a pair of negations if it satisfies the following conditions:

(N1) $n_{i}(\top)=\perp, n_{i}(\perp)=\top$ for all $i \in\{1,2\}$.

(N2) $n_{i}(x) \geq n_{i}(y)$ for $x \leq y$ and $i \in\{1,2\}$.

(N3) $n_{1}\left(n_{2}(x)\right)=n_{2}\left(n_{1}(x)\right)=x$ for all $x \in L$.

Definition 2.3.[10] A pair $\left(I_{1}, I_{2}\right)$ with maps $I_{1}, I_{2}: L \times L \rightarrow L$ is called a pair of implications if it satisfies the following conditions:

(I1) $I_{i}(\top, \top)=I_{i}(\perp, \top)=I_{i}(\perp, \perp)=\top, I_{i}(\top, \perp)=\perp$ for all $i \in\{1,2\}$.

(I2) If $x \leq y$, then $I_{i}(x, z) \geq I_{i}(y, z)$ for all $i \in\{1,2\}$.

(I3) $I_{i}(\top, x)=x$ for all $x \in L$ and $i \in\{1,2\}$.

A pair $\left(I_{1}, I_{2}\right)$ of implications is called a pair of E-implications if it satisfies the following exchange properties:

(E) $I_{1}\left(x, I_{2}(y, z)\right)=I_{2}\left(y, I_{1}(x, z)\right)$ for all $x, y, z \in L$.

A pair $\left(I_{1}, I_{2}\right)$ of implications is called a pair of S-implications if it satisfies the following strong properties:

(S) $I_{1}\left(I_{2}(x, \perp), \perp\right)=I_{2}\left(I_{1}(x, \perp), \perp\right)=x$.

A pair $\left(I_{1}, I_{2}\right)$ of implications is called a pair of SE-implications if it satisfies conditions (E) and (S).

In this paper, sometimes composition function $f \circ g \circ h$ will be denoted by fgh. 


\section{Pseudo t-Norms and Fuzzy Connectives}

Theorem 3.1. Let $T: L \times L \rightarrow L$ be a pseudo t-norm and $\left(n_{1}, n_{2}\right)$ a pair of negations on $L$. We define $T^{t}, S_{12}, S_{21}, I_{i}: L \times L \rightarrow L$

$$
\begin{gathered}
T^{t}(x, y)=T(y, x), \\
S_{12}(x, y)=n_{1}\left(T\left(n_{2}(x), n_{2}(y)\right), S_{21}(x, y)=n_{2}\left(T\left(n_{1}(x), n_{1}(y)\right) .\right.\right.
\end{gathered}
$$

The the following properties hold.

(1) $T^{t}$ is a pseudo t-norm.

(2) $S_{12}$ and $S_{21}$ are pseudo t-conorms.

(3) $S_{12}=S_{21}$ iff $S_{12}^{t}=S_{21}^{t}$ iff

$$
T(x, y)=n_{2} \circ n_{2}\left(T\left(n_{1}\left(n_{1}(x)\right), n_{1}\left(n_{1}(y)\right)\right)\right.
$$

where $S_{i j}^{t}(x, y)=S_{i j}(y, x)$.

(4) If $n_{1}=n_{2}$, then $S_{12}=S_{21}$ and $S_{12}^{t}=S_{21}^{t}$.

Proof (1) It is easily proved as a similar method as following (2).

(2) (S1) $S_{12}\left(S_{12}(x, y), z\right)=S_{12}\left(x, S_{12}(y, z)\right)$ from

$$
\begin{aligned}
& S_{12}\left(S_{12}(x, y), z\right) \\
& =n_{1}\left(T\left(n_{2}\left(S_{12}(x, y)\right), n_{2}(z)\right)\right) \\
& =n_{1}\left(T\left(n_{2} n_{1}\left(T\left(n_{2}(x), n_{2}(y)\right)\right), n_{2}(z)\right)\right) \\
& =n_{1}\left(T\left(T\left(n_{2}(x), n_{2}(y)\right), n_{2}(z)\right)\right) \\
& =n_{1}\left(T\left(n_{2}(x), T\left(n_{2}(y), n_{2}(z)\right)\right)\right), \\
& S_{12}\left(x, S_{12}(y, z)\right) \\
& =n_{1}\left(T\left(n_{2}(x), n_{2}\left(S_{12}(y, z)\right)\right)\right) \\
& \left.=n_{1}\left(T\left(n_{2}(x), n_{2} n_{1}\left(T\left(n_{2}(y), n_{2}(z)\right)\right)\right)\right)\right) \\
& =n_{1}\left(T\left(n_{2}(x), T\left(n_{2}(y), n_{2}(z)\right)\right)\right) .
\end{aligned}
$$

(S2) $S_{12}(x, \perp)=n_{1}\left(T\left(n_{2}(x), n_{2}(\perp)\right)=n_{1}\left(n_{2}(x)\right)=x\right.$. Similarly, $S_{12}(\perp, x)=$ $x$.

(S3) If $x \leq z$ and $y \leq w$, then $n_{2}(x) \geq n_{2}(z)$ and $n_{2}(y) \geq n_{2}(w)$. Thus $S_{12}(x, y) \leq \mathcal{S}_{12}(z, w)$.

Hence $S_{12}$ is a pseudo t-cornorm. Similarly, $S_{21}$ is a pseudo t-cornorm.

(3) It follows from:

$$
\begin{aligned}
& S_{12}(x, y)=S_{21}(x, y) \\
& \text { iff } n_{1}\left(T\left(n_{2}(x), n_{2}(y)\right)\right)=n_{2}\left(T\left(n_{1}(x), n_{1}(y)\right)\right) \\
& \text { iff } T\left(n_{2}(x), n_{2}(y)\right)=n_{2}\left(n_{2}\left(T\left(n_{1}(x), n_{1}(y)\right)\right)\right) \\
& \text { iff } T(x, y)=n_{2}\left(n_{2}\left(T\left(n_{1}\left(n_{1}(x)\right), n_{1}\left(n_{1}(y)\right)\right)\right)\right) \\
& \text { iff } S_{12}^{t}(x, y)=S_{21}^{t}(x, y) .
\end{aligned}
$$


(4) Since $n_{1}=n_{2}$, then $n_{1} n_{1}(x)=n_{2} n_{2}(x)=x$. In (3), it is trivial.

Example 3.2.Put $L=\left\{(x, y) \in R^{2} \mid(0,1) \leq(x, y) \leq(2,3)\right\}$ where $(0,1)$ is the bottom element and $(2,3)$ is the top element where

$$
\left(x_{1}, y_{1}\right) \leq\left(x_{2}, y_{2}\right) \Leftrightarrow y_{1}<y_{2} \text { or } y_{1}=y_{2}, x_{1} \leq x_{2} \text {. }
$$

(1) A map $T: L \times L \rightarrow L$ is defined as

$$
T\left(\left(x_{1}, y_{1}\right),\left(x_{2}, y_{2}\right)\right)=\left(x_{1}-\frac{2}{3} y_{1}+\frac{1}{3} x_{2} y_{1}, \frac{1}{3} y_{1} y_{2}\right) \vee(0,1) .
$$

(T1) $T\left(T\left(\left(x_{1}, y_{1}\right),\left(x_{2}, y_{2}\right)\right),\left(x_{3}, y_{3}\right)\right)=T\left(\left(x_{1}, y_{1}\right), T\left(\left(x_{2}, y_{2}\right),\left(x_{3}, y_{3}\right)\right)\right)$ from:

$$
\begin{aligned}
& T\left(T\left(\left(x_{1}, y_{1}\right),\left(x_{2}, y_{2}\right)\right),\left(x_{3}, y_{3}\right)\right) \\
& =T\left(\left(x_{1}-\frac{2}{3} y_{1}+\frac{1}{3} x_{2} y_{1}, \frac{1}{3} y_{1} y_{2}\right) \vee(0,1),\left(x_{3}, y_{3}\right)\right) \\
& =\left(x_{1}-\frac{2}{3} y_{1}+\frac{1}{3} x_{2} y_{1}-\frac{2}{9} y_{1} y_{2}+\frac{1}{3} y_{1} y_{2} x_{3},+\frac{1}{9} y_{1} y_{2} y_{3}\right) \vee(0,1) . \\
& T\left(\left(x_{1}, y_{1}\right), T\left(\left(x_{2}, y_{2}\right),\left(x_{3}, y_{3}\right)\right)\right) \\
& =T\left(\left(x_{1}, y_{1}\right),\left(x_{2}-\frac{2}{3} y_{2}+\frac{1}{3} x_{3} y_{2}, \frac{1}{3} y_{2} y_{3}\right) \vee(0,1)\right) \\
& =\left(x_{1}-\frac{2}{3} y_{1}+\frac{1}{3} x_{2} y_{1}-\frac{2}{9} y_{1} y_{2}+\frac{1}{3} y_{1} y_{2} x_{3},+\frac{1}{9} y_{1} y_{2} y_{3}\right) \vee(0,1) .
\end{aligned}
$$

(T2) If $\left(x_{1}, y_{1}\right) \leq\left(x_{2}, y_{2}\right)$, then $y_{1}<y_{2}$ or $y_{1}=y_{2}, x_{1} \leq x_{2}$. Thus

$$
\begin{aligned}
& T\left(\left(x_{1}, y_{1}\right),\left(x_{3}, y_{3}\right)\right)=\left(x_{1}-\frac{2}{3} y_{1}+\frac{1}{3} x_{3} y_{1}, \frac{1}{3} y_{1} y_{3}\right) \vee(0,1) \\
& \leq\left(x_{2}-\frac{2}{3} y_{2}+\frac{1}{3} x_{3} y_{2}, \frac{1}{3} y_{2} y_{3}\right) \vee(0,1)=T\left(\left(x_{2}, y_{2}\right),\left(x_{3}, y_{3}\right)\right) .
\end{aligned}
$$

(T3)

$$
T\left(\left(x_{1}, y_{1}\right),(2,3)\right)=\left(x_{1}, y_{1}\right)=T\left((2,3),\left(x_{1}, y_{1}\right)\right) .
$$

Then $T$ is a pseudo t-norm but not t-norm because

$$
\left(1, \frac{4}{3}\right)=T((3,2),(-1,2)) \neq T((-1,2),(3,2))=\left(-\frac{1}{3}, \frac{4}{3}\right) .
$$

(2) We obtain a pair $\left(n_{1}, n_{2}\right)$ of negations where

$$
\begin{gathered}
n_{1}(x, y)=\left(2-\frac{3 x}{y}, \frac{3}{y}\right), \quad n_{2}(x, y)=\left(\frac{2-x}{y}, \frac{3}{y}\right) . \\
n_{1} n_{2}(x, y)=(x, y), \quad n_{2} n_{1}(x, y)=(x, y) .
\end{gathered}
$$

(3) By Theorem 3.1(2), we obtain

$$
\begin{aligned}
& \left.S_{12}\left(\left(x_{1}, y_{1}\right),\left(x_{2}, y_{2}\right)\right)=n_{1} T\left(n_{2}\left(x_{1}, y_{1}\right), n_{2}\left(x_{2}, y_{2}\right)\right)\right) \\
& \left.=n_{1} T\left(\left(\frac{2-x_{1}}{y_{1}}, \frac{3}{y_{1}}\right),\left(\frac{2-x_{2}}{y_{2}}, \frac{3}{y_{2}}\right)\right)=n_{1}\left(\frac{2-x_{1}}{y_{1}}-\frac{2}{y_{1}}+\frac{2-x_{2}}{y_{1} y_{2}}, \frac{3}{y_{1} y_{2}}\right)\right) \wedge(2,3) \\
& =\left(x_{2}+x_{1} y_{2}, y_{1} y_{2}\right) \wedge(2,3),
\end{aligned}
$$




$$
\begin{aligned}
& \left.S_{21}\left(\left(x_{1}, y_{1}\right),\left(x_{2}, y_{2}\right)\right)=n_{2} T\left(n_{1}\left(x_{1}, y_{1}\right), n_{1}\left(x_{2}, y_{2}\right)\right)\right) \\
& \left.=n_{2} T\left(\left(2-\frac{3 x_{1}}{y_{1}}, \frac{3}{y_{1}}\right),\left(2-\frac{3 x_{2}}{y_{2}}, \frac{3}{y_{2}}\right)\right)=n_{2}\left(2-\frac{3 x_{1}}{y_{1}}-\frac{3 x_{2}}{y_{1} y_{2}}, \frac{3}{y_{1} y_{2}}\right)\right) \wedge(2,3) \\
& =\left(x_{2}+x_{1} y_{2}, y_{1} y_{2}\right) \wedge(2,3) .
\end{aligned}
$$

(4) Since

$$
\begin{aligned}
& n_{1} n_{1}(x, y)=(3 x-2 y+2, y), \quad n_{2} n_{2}(x, y)=\left(\frac{x+2 y-2}{3}, y\right) \\
& \quad n_{2} n_{2}\left(T\left(n_{1}\left(n_{1}\left(x_{1}, y_{1}\right)\right), n_{1}\left(n_{1}\left(x_{2}, y_{2}\right)\right)\right)\right) \\
& \quad=n_{2} n_{2}\left(T\left(\left(3 x_{1}-2 y_{1}+2, y_{1}\right),\left(3 x_{2}-2 y_{2}+2, y_{2}\right)\right),\right. \\
& \quad=n_{2} n_{2}\left(\left(\frac{1}{3}\left(3 x_{1}-2 y_{1}+x_{2} y_{1}\right), \frac{1}{3} y_{1} y_{2}\right) \vee(0,1)\right), \\
& \left.\quad=\left(x_{1}-\frac{2}{3} y_{1}+\frac{1}{3} x_{2} y_{1}, \frac{1}{3} y_{1} y_{2}\right) \vee(0,1)\right) \\
& \quad=T\left(\left(x_{1}, y_{1}\right),\left(x_{2}, y_{2}\right)\right) .
\end{aligned}
$$

By Theorem $3.1(3), S_{12}=S_{21}$ and $S_{12}^{t}=S_{21}^{t}$.

Theorem 3.3. Let $T: L \times L \rightarrow L$ be a pseudo t-norm and $\left(n_{1}, n_{2}\right)$ a pair of negations on $L$. We define $I_{i}: L \times L \rightarrow L$

$$
\begin{gathered}
I_{1}(x, y)=n_{1}\left(T\left(x, n_{2}(y)\right)\right), \quad I_{2}(x, y)=n_{1}\left(T\left(n_{2}(y), x\right)\right), \\
I_{3}(x, y)=n_{2}\left(T\left(x, n_{1}(y)\right)\right), \quad I_{4}(x, y)=n_{2}\left(T\left(n_{1}(y), x\right)\right), \\
I_{5}(x, y)=n_{1}\left(T \left(n_{2}\left(y, n_{2}\left(n_{2}(x)\right)\right), I_{6}(x, y)=n_{2}\left(T\left(n_{1}(y), n_{1}\left(n_{1}(x)\right)\right),\right.\right.\right. \\
I_{7}(x, y)=n_{1}\left(T\left(n_{2} n_{2}(x), n_{2}(y)\right)\right), I_{8}(x, y)=n_{2}\left(T\left(n_{1} n_{1}(x), n_{1}(y)\right)\right) .
\end{gathered}
$$

The the following properties hold.

(1) The pair $\left(I_{1}, I_{2}\right)$ is a pair of $E$-implications such that

$$
\begin{gathered}
I_{1}(T(x, y), z)=I_{1}\left(x, I_{1}(y, z)\right), \\
I_{2}(T(x, y), z)=I_{2}\left(y, I_{2}(x, z)\right), \\
I_{1}\left(I_{2}(x, \perp), \perp\right)=I_{2}\left(I_{1}(x, \perp), \perp\right)=n_{1}\left(n_{1}(x)\right) .
\end{gathered}
$$

(2) If $n_{1}=n_{2}$, then $\left(I_{1}, I_{2}\right)$ is a pair of $S E$-implications.

(3) If $x \leq y$ iff $I_{1}(x, y)=\top$ iff $I_{2}(x, y)=\top$, then $T(x, y) \leq z$ iff $x \leq I_{1}(y, z)$ iff $y \leq I_{2}(x, z)$.

(4) The pair $\left(I_{3}, I_{4}\right)$ is a pair of $E$-implications such that

$$
\begin{aligned}
& I_{3}(T(x, y), z)=I_{3}\left(x, I_{3}(y, z)\right), \\
& I_{4}(T(x, y), z)=I_{4}\left(y, I_{4}(x, z)\right)
\end{aligned}
$$




$$
I_{3}\left(I_{4}(x, \perp), \perp\right)=I_{4}\left(I_{3}(x, \perp), \perp\right)=n_{2}\left(n_{2}(x)\right) .
$$

(5) If $n_{1}=n_{2}$, then $\left(I_{3}, I_{4}\right)$ is a pair of implications.

(6) If $x \leq y$ iff $I_{3}(x, y)=\top$ iff $I_{4}(x, y)=\top$, then $T(x, y) \leq z$ iff $x \leq I_{3}(y, z)$ iff $y \leq I_{4}(x, z)$.

(7) The pair $\left(I_{2}, I_{3}\right)$ is a pair of $S$-implications.

(8) If it satisfies the following condition

$$
\begin{aligned}
& T\left(x, n_{1} n_{1}\left(T\left(n_{2}\left(n_{2} y, z\right)\right)\right)\right. \\
& \left.=n_{1} n_{1}\left(T\left(n_{2}\left(n_{2}(T(x, y))\right)\right), z\right)\right),
\end{aligned}
$$

then $\left(I_{2}, I_{3}\right)$ is a pair of $S E$-implications and if $x \leq y$ iff $I_{2}(x, y)=\top$ iff $I_{3}(x, y)=\top$, then $T(x, y) \leq z$ iff $x \leq I_{3}(y, z)$ iff $y \leq I_{2}(x, z)$.

(9) The pair $\left(I_{1}, I_{4}\right)$ is a pair of $S$-implications.

(10) If it satisfies the following condition

$$
T\left(x, n_{2} n_{2}\left(T\left(n_{1}\left(n_{1}(y)\right), z\right)\right)\right)=n_{2} n_{2}\left(T\left(n_{1}\left(n_{1}(T(x, y))\right), z\right)\right),
$$

then $\left(I_{1}, I_{4}\right)$ is a pair of $S E$-implications and if $x \leq y$ iff $I_{1}(x, y)=\top$ iff $I_{4}(x, y)=\top$, then $T(x, y) \leq z$ iff $x \leq I_{1}(y, z)$ iff $y \leq I_{4}(x, z)$.

(11) If $n_{1}=n_{2}$, then $\left(I_{2}, I_{3}\right)$ and $\left(I_{1}, I_{4}\right)$ are pairs of $S E$-implications.

(12) The pair $\left(I_{1}, I_{5}\right)$ is a pair of $S E$-implications. If $x \leq y$ iff $I_{1}(x, y)=\top$ iff $I_{5}(x, y)=\top$, then $T(x, y) \leq z$ iff $x \leq I_{1}(y, z)$ iff $y \leq I_{5}(x, z)$.

(13) The pair $\left(I_{3}, I_{6}\right)$ is a pair of $S E$-implications. If $x \leq y$ iff $I_{3}(x, y)=\top$ iff $I_{6}(x, y)=\top$, then $T(x, y) \leq z$ iff $x \leq I_{3}(y, z)$ iff $y \leq I_{6}(x, z)$.

(14) The pair $\left(I_{2}, I_{7}\right)$ is a pair of $S E$-implications. If $x \leq y$ iff $I_{2}(x, y)=\top$ iff $I_{7}(x, y)=\top$, then $T(x, y) \leq z$ iff $x \leq I_{7}(y, z)$ iff $y \leq I_{2}(x, z)$.

(15) The pair $\left(I_{4}, I_{8}\right)$ is a pair of $S E$-implications. If $x \leq y$ iff $I_{4}(x, y)=\top$ iff $I_{8}(x, y)=\top$, then $T(x, y) \leq z$ iff $x \leq I_{8}(y, z)$ iff $y \leq I_{4}(x, z)$.

Proof (1) (I1) Since $T(\top, \top)=\top$ and $T(\top, \perp)=T(\perp, \top)=T(\perp, \perp)=\perp$ , we have

$$
I_{i}(\top, \perp)=\perp, I_{i}(\top, \top)=I_{i}(\perp, \top)=I_{i}(\perp, \perp)=\top .
$$

(I2) If $x \geq z$ and $y \leq w$, then $n_{i}(x) \leq n_{i}(z)$ and $n_{i}(y) \geq n_{i}(w)$, for $i=1,2$. Thus, for $i=1,2$,

$$
I_{i}(x, y) \leq I_{i}(z, w)
$$

(I3) Since $I_{1}(\top, x)=n_{1}\left(T\left(\top, n_{2}(x)\right)\right)=n_{1} n_{2}(x)=x$ and $I_{2}(\top, x)=$ $n_{1}\left(T\left(n_{2}(x), \top\right)\right)=n_{1} n_{2}(x)=x$. 
(I4) $I_{2}\left(x, I_{1}(y, z)\right)=I_{1}\left(y, I_{2}(x, z)\right)$ from:

$$
\begin{aligned}
& I_{2}\left(x, I_{1}(y, z)\right)=n_{1}\left(T\left(n_{2}\left(I_{1}(y, z)\right), x\right)\right) \\
& =n_{1}\left(T\left(n_{2} n_{1}\left(T\left(y, n_{2}(z)\right)\right), x\right)\right)=n_{1}\left(T\left(T\left(y, n_{2}(z)\right), x\right)\right) \\
& I_{1}\left(y, I_{2}(x, z)\right)=n_{1}\left(T\left(y, n_{2}\left(I_{2}(x, z)\right)\right)\right) \\
& =n_{1}\left(T\left(y, n_{2} n_{1} T\left(n_{2}(z), x\right)\right)=n_{1}\left(T\left(y, T\left(n_{2}(z), x\right)\right) .\right.\right.
\end{aligned}
$$

Thus, $\left(I_{1}, I_{2}\right)$ is a pair of $E$-implications. Since $T\left(x, n_{2}(y)\right)=n_{2}\left(I_{1}(x, y)\right)$, we have

$$
\begin{aligned}
& I_{1}(T(x, y), z)=n_{1}\left(T\left(T(x, y), n_{2}(z)\right)\right) \\
& =n_{1}\left(T\left(T(x, y), n_{2}(z)\right)\right)=n_{1}\left(T\left(x, T\left(y, n_{2}(z)\right)\right)\right) \\
& =n_{1}\left(T\left(x, n_{2}\left(I_{1}(y, z)\right)\right)=I_{1}\left(x, I_{1}(y, z)\right),\right. \\
& I_{2}(T(x, y), z)=n_{1}\left(T\left(n_{2}(z), T(x, y)\right)\right) \\
& =n_{1}\left(T\left(n_{2}(z), T(x, y)\right)\right)=n_{1}\left(T\left(T\left(n_{2}(z), x\right), y\right)\right) \\
& =n_{1}\left(T\left(n_{2}\left(I_{2}(x, z)\right), y\right)\right)=I_{2}\left(y, I_{2}(x, z)\right) . \\
& \\
& I_{1}(x, \perp)=n_{1}\left(T\left(x, n_{2}(\perp)\right)\right)=n_{1}(x)=I_{2}(x, \perp) . \\
& I_{1}\left(I_{2}(x, \perp), \perp\right)=I_{1}\left(I_{2}(x, \perp), \perp\right)=n_{1}\left(n_{1}(x)\right) .
\end{aligned}
$$

(2) If $n_{1}=n_{2}$, then $n_{1} n_{1}(x)=n_{2} n_{2}(x)=x$. Thus

$$
I_{1}\left(I_{2}(x, \perp), \perp\right)=n_{1} n_{1}(x)=x .
$$

Hence $\left(I_{1}, I_{2}\right)$ is a pair of implications.

(3) From (1), since

$$
\begin{aligned}
& I_{1}(T(x, y), z)=I_{1}\left(x, \mathcal{I}_{1}(y, z)\right), \\
& I_{2}(T(x, y), z)=\mathcal{I}_{2}\left(y, I_{2}(x, z)\right),
\end{aligned}
$$

then $T(x, y) \leq z$ iff $x \leq I_{1}(y, z)$ iff $y \leq I_{2}(x, z)$.

(4)-(6) are similarly proved as (1)-(3), respectively.

(7) It is easily proved from

$$
\begin{aligned}
& I_{2}(x, \perp)=n_{1}\left(T\left(n_{2}(\perp), x\right)\right)=n_{1}(x) \\
& I_{3}(x, \perp)=n_{2}\left(T\left(x, n_{1}(\perp)\right)\right)=n_{2}(x) \\
& I_{2}\left(I_{3}(x, \perp), \perp\right)=n_{1}\left(n_{2}(x)\right)=x, \\
& I_{2}\left(I_{3}(x, \perp), \perp\right)=n_{2}\left(n_{1}(x)\right)=x .
\end{aligned}
$$


(8)

$$
\begin{aligned}
& I_{3}\left(x, I_{2}(y, z)\right) \\
& =n_{2}\left(T\left(x, n_{1}\left(I_{2}(y, z)\right)\right)\right) \\
& =n_{2}\left(T\left(x, n_{1}\left(n_{1}\left(T\left(n_{2}(z), y\right)\right)\right)\right)\right) \\
& =n_{2}\left(T\left(x, n_{1}\left(n_{1}\left(T\left(n_{2}(z), y\right)\right)\right)\right)\right) \\
& I_{2}\left(y, I_{3}(x, z)\right) \\
& =n_{1}\left(T\left(n_{2}\left(\left(I_{3}(x, z)\right)\right), y\right)\right) \\
& =n_{1}\left(T\left(n_{2}\left(n_{2}\left(T\left(x, n_{1}(z)\right)\right)\right), y\right)\right. \\
& =n_{1}\left(T\left(n_{2}\left(n_{2}\left(T\left(x, n_{1}(z)\right)\right)\right), y\right)\right)
\end{aligned}
$$

Hence $I_{3}\left(x, I_{2}(y, z)\right)=I_{2}\left(y, I_{3}(x, z)\right)$ iff

$$
\begin{aligned}
& T\left(x, n_{1} n_{1}\left(T\left(n_{2}\left(n_{2} y, z\right)\right)\right)\right. \\
& \left.=n_{1} n_{1}\left(T\left(n_{2}\left(n_{2}(T(x, y))\right)\right), z\right)\right) .
\end{aligned}
$$

It follows that $\left(I_{2}, I_{3}\right)$ is a pair of implications.

(9) and (10) are similarly proved as (7) and (8), respectively.

(11) It is trivial from $n_{1}\left(n_{1}(x)\right)=x=n_{2}\left(n_{2}(x)\right)$, (12) and (14).

(12) $I_{5}\left(x, I_{1}(y, z)\right)=I_{1}\left(y, I_{5}(x, z)\right)$ from:

$$
\begin{aligned}
& I_{5}\left(x, I_{1}(y, z)\right) \\
& =n_{1}\left(T\left(n_{2}\left(I_{1}(y, z), n_{2} n_{2}(x)\right)\right)\right. \\
& \left.=n_{1}\left(T\left(n_{2} n_{1}\left(T\left(y, n_{2}(z)\right)\right)\right), n_{2} n_{2}(x)\right)\right) \\
& =n_{1}\left(T\left(T\left(y, n_{2}(z)\right)\right), n_{2} n_{2}(x)\right) \\
& I_{1}\left(y, I_{5}(x, z)\right) \\
& =n_{1}\left(T\left(y, n_{2}\left(I_{5}(x, z)\right)\right)\right) \\
& =n_{1}\left(T\left(y, n_{2} n_{1} T\left(n_{2}(z), n_{2} n_{2} x\right)\right)\right. \\
& =n_{1}\left(T\left(y, T\left(n_{2}(z), n_{2} n_{2} x\right)\right) .\right.
\end{aligned}
$$

Since

$$
I_{1}(x, \perp)=n_{1}\left(T\left(x, n_{2}(\perp)\right)\right)=n_{1}(x)
$$

and

$$
\begin{gathered}
I_{5}(x, \perp)=n_{1}\left(T\left(n_{2}(\perp), n_{2} n_{2}(x)\right)\right)=n_{1} n_{2} n_{2}(x)=n_{2}(x), \\
I_{1}\left(I_{5}(x, \perp), \perp\right)=n_{1} n_{2}(x)=x . \\
I_{5}\left(I_{1}(x, \perp), \perp\right)=n_{2} n_{1}(x)=x .
\end{gathered}
$$

(13) It is similarly proved as (12). 
(14) $I_{7}\left(x, I_{2}(y, z)\right)=I_{2}\left(y, I_{7}(x, z)\right)$ from:

$$
\begin{aligned}
& I_{7}\left(x, I_{2}(y, z)\right) \\
& =n_{1}\left(T\left(n_{2} n_{2}(x), n_{2}\left(I_{2}(y, z)\right)\right)\right. \\
& \left.=n_{1}\left(T\left(n_{2} n_{2}(x), n_{2} n_{1}\left(T\left(n_{2}(z), y\right)\right)\right)\right)\right) \\
& =n_{1}\left(T\left(n_{2} n_{2}(x), T\left(n_{2}(z), y\right)\right)\right) \\
& I_{2}\left(y, I_{7}(x, z)\right) \\
& =n_{1}\left(T\left(n_{2}\left(I_{7}(x, z)\right), y\right)\right) \\
& =n_{1}\left(T\left(n_{2} n_{1} T\left(n_{2} n_{2} x, n_{2}(z)\right), y\right)\right) \\
& =n_{1}\left(T\left(T\left(n_{2} n_{2} x, n_{2}(z)\right), y\right)\right) .
\end{aligned}
$$

Since

$$
I_{2}(x, \perp)=n_{1}\left(T\left(n_{2}(\perp), x\right)\right)=n_{1}(x)
$$

and

$$
\begin{gathered}
I_{7}(x, \perp)=n_{1}\left(T\left(n_{2}(\perp), n_{2} n_{2}(x)\right)\right)=n_{1} n_{2} n_{2}(x)=n_{2}(x), \\
I_{2}\left(I_{7}(x, \perp), \perp\right)=n_{1} n_{2}(x)=x . \\
I_{7}\left(I_{2}(x, \perp), \perp\right)=n_{2} n_{1}(x)=x .
\end{gathered}
$$

(15) It is similarly proved as (14).

Example 3.4.Put $L=\left\{(x, y) \in R^{2} \mid(0,1) \leq(x, y) \leq(2,3)\right\}, T$ a pseudo t-norm and $\left(n_{1}, n_{2}\right)$ be a pair of negations in Example 3.2 .

(1)

$$
\begin{aligned}
& I_{1}\left(\left(x_{1}, y_{1}\right),\left(x_{2}, y_{2}\right)\right)=n_{1} T\left(\left(x_{1}, y_{1}\right), n_{2}\left(x_{2}, y_{2}\right)\right) \\
&=n_{1} T\left(\left(x_{1}, y_{1}\right),\left(\frac{2-x_{2}}{y_{2}}, \frac{3}{y_{2}}\right)\right) \\
&=n_{1}\left(x_{1}-\frac{2}{3} y_{1}+\frac{2 y_{1}-x_{2} y_{1}}{3 y_{2}}, \frac{y_{1}}{y_{2}}\right) \wedge(2,3) \\
&=\left(x_{2}+2 y_{2}-\frac{3 x_{1} y_{2}}{y_{1}}, \frac{3 y_{2}}{y_{1}}\right) \wedge(2,3) . \\
& I_{2}\left(\left(x_{1}, y_{1}\right),\left(x_{2}, y_{2}\right)\right)=n_{1} T\left(n_{2}\left(x_{2}, y_{2}\right),\left(x_{1}, y_{1}\right)\right) \\
&= n_{1} T\left(\left(\frac{2-x_{2}}{y_{2}}, \frac{3}{y_{2}}\right),\left(x_{1}, y_{1}\right)\right)=n_{1}\left(\frac{x_{1}-x_{2}}{y_{2}}, \frac{y 1}{y_{2}}\right) \wedge(2,3) \\
&=\left(2-\frac{3\left(x_{1}-x_{2}\right.}{y_{1}}, \frac{3 y_{2}}{y_{1}}\right) \wedge(2,3) . \\
& I_{3}\left(\left(x_{1}, y_{1}\right),\left(x_{2}, y_{2}\right)\right)=n_{2} T\left(\left(x_{1}, y_{1}\right), n_{1}\left(x_{2}, y_{2}\right)\right) \\
&=n_{2} T\left(\left(x_{1}, y_{1}\right),\left(2-\frac{3 x_{2}}{y_{2}}, \frac{3}{y_{2}}\right)\right)=n_{2}\left(x_{1}-\frac{x_{2} y_{1}}{y_{2}}, \frac{y_{1}}{y_{2}}\right) \wedge(2,3) \\
&=\left(x_{2}+\frac{\left(2-x_{1}\right) y_{2}}{y_{1}}, \frac{3 y_{2}}{y_{1}}\right) \wedge(2,3) . \\
& \quad I_{4}\left(\left(x_{1}, y_{1}\right),\left(x_{2}, y_{2}\right)\right)=n_{2} T\left(n_{1}\left(x_{2}, y_{2}\right),\left(x_{1}, y_{1}\right)\right) \\
&=n_{2} T\left(\left(2-\frac{3 x_{2}}{y_{2}}, \frac{3}{y_{2}}\right),\left(x_{1}, y_{1}\right)\right) \\
&=n_{2}\left(2-\frac{3 x_{2}}{y_{2}}-\frac{2}{y_{2}}+\frac{x_{1}}{y_{2}}, \frac{y 1}{y_{2}}\right) \wedge(2,3) \\
&=\left(\frac{3 x_{2}-x_{1}+2}{y_{1}}, \frac{3 y_{2}}{y_{1}}\right) \wedge(2,3) .
\end{aligned}
$$




$$
\begin{aligned}
& I_{5}\left(\left(x_{1}, y_{1}\right),\left(x_{2}, y_{2}\right)\right)=n_{1} T\left(n_{2}\left(x_{2}, y_{2}\right), n_{2} n_{2}\left(x_{1}, y_{1}\right)\right) \\
& =n_{1} T\left(\left(\frac{2-x_{2}}{y_{2}}, \frac{3}{y_{2}}\right),\left(\frac{1}{3}\left(x_{1}+2 y_{1}-2\right), y_{1}\right)\right) \\
& =n_{1}\left(\frac{-x_{2}}{y_{2}}+\frac{x_{1}+2 y_{1}-2}{3 y_{2}}, \frac{y_{1}}{y_{2}}\right) \wedge(2,3) \\
& =\left(\frac{3 x_{2}-x_{1}+2}{y_{1}}, \frac{3 y_{2}}{y_{1}}\right) \wedge(2,3) . \\
& I_{6}\left(\left(x_{1}, y_{1}\right),\left(x_{2}, y_{2}\right)\right)=n_{2} T\left(n_{1}\left(x_{2}, y_{2}\right), n_{1} n_{1}\left(x_{1}, y_{1}\right)\right) \\
& =n_{2} T\left(\left(2-\frac{3 x_{2}}{y_{2}}, \frac{3}{y_{2}}\right),\left(3 x_{1}-2 y_{1}+2, y_{1}\right)\right) \\
& =n_{2}\left(2-\frac{3 x_{2}-3 x_{1}+2 y_{1}}{y_{2}}, \frac{y_{1}}{y_{2}}\right) \wedge(2,3) \\
& =\left(\frac{3 x_{2}-3 x_{1}+2 y_{1}}{y_{1}}, \frac{3 y_{2}}{y_{1}}\right) \wedge(2,3) . \\
& I_{7}\left(\left(x_{1}, y_{1}\right),\left(x_{2}, y_{2}\right)\right)=n_{1} T\left(n_{2} n_{2}\left(x_{1}, y_{1}\right), n_{2}\left(x_{2}, y_{2}\right)\right) \\
& =n_{1} T\left(\left(\frac{x_{1}+2 y_{1}-2}{3}, y_{1}\right),\left(\frac{2-x_{2}}{y_{2}}, \frac{3}{y_{2}}\right)\right) \\
& =n_{1}\left(\frac{x_{1}-2}{3}+\frac{\left(2-x_{2}\right) y_{1}}{3 y_{2}}, \frac{y_{1}}{y_{2}}\right) \wedge(2,3) \\
& =\left(x_{2}-\frac{\left(x_{1}-2\right) y_{2}}{y_{1}}, \frac{3 y_{2}}{y_{1}}\right) \wedge(2,3) . \\
& I_{8}\left(\left(x_{1}, y_{1}\right),\left(x_{2}, y_{2}\right)\right)=n_{2} T\left(n_{1} n_{1}\left(x_{1}, y_{1}\right), n_{1}\left(x_{2}, y_{2}\right)\right) \\
& =n_{2} T\left(\left(3 x_{1}-2 y_{1}+2, y_{1}\right),\left(2-\frac{3 x_{2}}{y_{2}}, \frac{3}{y_{2}}\right)\right) \\
& =n_{2}\left(3 x_{1}-2 y_{1}+2-\frac{x_{2} y_{1}}{y_{2}}, \frac{y_{1}}{y_{2}}\right) \wedge(2,3) \\
& =\left(x_{2}+2 y_{2}-\frac{3 x_{1} y_{2}}{y_{1}}, \frac{3 y_{2}}{y_{1}}\right) \wedge(2,3) .
\end{aligned}
$$

(2) A pair $\left(I_{1}, I_{2}\right)$ is a pair of $E$-implications, but not a pair of $S E$-implications

$$
I_{1}\left(I_{2}((2,2),(0,1)),(0,1)\right)=n_{1} n_{1}(2,2)=(4,2) \neq(2,2) .
$$

By Theorem $3.1(3)$, since $I_{1}((2,2),(1,2))=(2,3)$ but $(2,2) \not \leq(1,2)$, it does not satisfy the conditions in Theorem 3.1(3). Moreover,

$$
\begin{gathered}
(2,2)=T((2,3),(2,2)) \not \leq(1,2), \\
I_{1}(T((2,3),(2,2)),(1,2))=I_{1}\left((2,3), I_{1}((2,2),(2,1))=(2,3) .\right.
\end{gathered}
$$

(3)

$$
\begin{aligned}
& T\left(\left(x_{1}, y_{1}\right), n_{1} n_{1}\left(T\left(n_{2} n_{2}\left(x_{2}, y_{2}\right),\left(x_{3}, y_{3}\right)\right)\right)\right) \\
& =T\left(\left(x_{1}, y_{1}\right), n_{1} n_{1}\left(T\left(\frac{x_{2}+2 y_{2}-2}{3}, y_{2}\right),\left(x_{3}, y_{3}\right)\right)\right) \\
& =T\left(\left(x_{1}, y_{1}\right), n_{1} n_{1}\left(\frac{x_{2}+2 y_{2}-2}{3}-\frac{2}{3} y_{2}+\frac{1}{3} x_{3} y_{2}, \frac{1}{3} y_{2} y_{3}\right) \wedge(1,0)\right) \\
& =T\left(\left(x_{1}, y_{1}\right),\left(x_{2}+x_{3} y_{2}-\frac{2}{3} y_{3} y_{2}, \frac{1}{3} y_{2} y_{3}\right) \wedge(1,0)\right) \\
& =\left(x_{1}+\frac{1}{3} x_{2} y_{1}-\frac{2}{3} y_{1}+\frac{1}{3} x_{3} y_{2} y_{1}-\frac{2}{9} y_{3} y_{2} y_{1}, \frac{1}{9} y_{1} y_{2} y_{3}\right) \wedge(1,0) .
\end{aligned}
$$




$$
\begin{aligned}
& n_{1} n_{1}\left(T\left(n_{2} n_{2} T\left(\left(x_{1}, y_{1}\right),\left(x_{2}, y_{2}\right)\right),\left(x_{3}, y_{3}\right)\right)\right) \\
& \left.=n_{1} n_{1}\left(T\left(n_{2} n_{2}\left(x_{1}-\frac{2}{3} y_{1}+\frac{1}{3} x_{2} y_{1}\right), \frac{1}{3} y_{2} y_{1}\right),\left(x_{3}, y_{3}\right)\right)\right) \\
& \left.=n_{1} n_{1}\left(T\left(\frac{\left(x_{1}-\frac{2}{3} y_{1}+\frac{1}{3} x_{2} y_{1}+\frac{2}{3} y_{2} y_{1}-2\right)}{3}, \frac{1}{3} y_{2} y_{1}\right),\left(x_{3}, y_{3}\right)\right)\right) \\
& =\left(x_{1}+\frac{1}{3} x_{2} y_{1}-\frac{2}{3} y_{1}+\frac{1}{3} x_{3} y_{2} y_{1}-\frac{2}{9} y_{3} y_{2} y_{1}, \frac{1}{9} y_{1} y_{2} y_{3}\right) \wedge(1,0) .
\end{aligned}
$$

Since

$$
\begin{aligned}
& T\left(\left(x_{1}, y_{1}\right), n_{1} n_{1}\left(T\left(n_{2} n_{2}\left(x_{2}, y_{2}\right),\left(x_{3}, y_{3}\right)\right)\right)\right) \\
& =n_{1} n_{1}\left(T\left(n_{2} n_{2} T\left(\left(x_{1}, y_{1}\right),\left(x_{2}, y_{2}\right)\right),\left(x_{3}, y_{3}\right)\right)\right),
\end{aligned}
$$

by Theorem 3.3(8), $\left(I_{2}, I_{3}\right)$ is a pair of $S E$-implications ;i.e.

$$
\begin{aligned}
& I_{2}\left(\left(x_{1}, y_{1}\right), I_{3}\left(\left(x_{2}, y_{2}\right),\left(x_{3}, y_{3}\right)\right)\right) \\
& =I_{3}\left(\left(x_{2}, y_{2}\right), I_{2}\left(\left(x_{1}, y_{1}\right),\left(x_{3}, y_{3}\right)\right)\right) .
\end{aligned}
$$

Moreover, since $\left(x_{1}, y_{1}\right) \leq\left(x_{2}, y_{2}\right)$ iff $y_{1}<y_{2}$ or $y_{1}=y_{2}, x_{1} \leq x_{2}$ iff $I_{2}\left(\left(x_{1}, y_{1}\right),\left(x_{2}, y_{2}\right)\right)=(2,3)$ iff $I_{3}\left(\left(x_{1}, y_{1}\right),\left(x_{2}, y_{2}\right)\right)=(2,3)$, then $T\left(\left(x_{1}, y_{1}\right)\right.$, $\left.\left(x_{2}, y_{2}\right)\right) \leq\left(x_{3}, y_{3}\right)$ iff $\left(x_{1}, y_{1}\right) \leq I_{3}\left(\left(x_{2}, y_{2}\right),\left(x_{3}, y_{3}\right)\right)$ iff $\left(x_{2}, y_{2}\right) \leq I_{2}\left(\left(x_{1}, y_{1}\right)\right.$, $\left.\left(x_{3}, y_{3}\right)\right)$.

(4) A pair $\left(I_{3}, I_{4}\right)$ is a pair of $E$-implications, but not a pair of $S E$-implications

$$
I_{3}\left(I_{4}((2,2),(0,1)),(0,1)\right)=n_{2} n_{2}(2,2)=\left(\frac{4}{3}, 2\right) \neq(2,2) .
$$

By Theorem $3.3(8)$, since $I_{4}\left(\left(\frac{3}{2}, \frac{3}{2}\right),\left(1, \frac{3}{2}\right)\right)=(2,3)$ but $\left(\frac{3}{2}, \frac{3}{2}\right) \not \subset\left(1, \frac{3}{2}\right)$, it does not satisfy the conditions in Theorem 3.3(6). Moreover,

$$
\begin{gathered}
T\left((2,3),\left(\frac{3}{2}, \frac{3}{2}\right)\right) \not \leq\left(1, \frac{3}{2}\right), \\
I_{4}\left(T\left((2,3),\left(\frac{3}{2}, \frac{3}{2}\right)\right),\left(1, \frac{3}{2}\right)\right)=I_{4}\left((2,3), I_{4}\left(\left(\frac{3}{2}, \frac{3}{2}\right),\left(1, \frac{3}{2}\right)\right)=(2,3) .\right.
\end{gathered}
$$

(5) Since

$$
\begin{aligned}
& T\left(\left(x_{1}, y_{1}\right), n_{2} n_{2}\left(T\left(n_{1} n_{1}\left(x_{2}, y_{2}\right),\left(x_{3}, y_{3}\right)\right)\right)\right) \\
& =T\left(\left(x_{1}, y_{1}\right), n_{2} n_{2}\left(3 x_{2}-2 y_{2}+2-\frac{2}{3} y_{2}+\frac{1}{3} x_{3} y_{2}, \frac{1}{3} x_{3} y_{2}\right)\right) \\
& =\left(x_{1}-\frac{2}{3} y_{1}+\frac{1}{3} x_{2} y_{1}-\frac{8}{27} y_{1} y_{2}+\frac{1}{27} x_{3} y_{1} y_{2}+\frac{2}{27} y_{1} y_{2} y_{3}, \frac{1}{9} y_{1} y_{2} y_{3}\right) \vee(0,1) \\
& =n_{2} n_{2}\left(T\left(n_{1} n_{1} T\left(\left(x_{1}, y_{1}\right),\left(x_{2}, y_{2}\right)\right),\left(x_{3}, y_{3}\right)\right)\right),
\end{aligned}
$$

by Theorem 3.3(10), $\left(I_{1}, I_{4}\right)$ is a pair of $S E$-implications ;i.e.

$$
\begin{aligned}
& I_{1}\left(\left(x_{1}, y_{1}\right), I_{4}\left(\left(x_{2}, y_{2}\right),\left(x_{3}, y_{3}\right)\right)\right) \\
& =I_{4}\left(\left(x_{2}, y_{2}\right), I_{1}\left(\left(x_{1}, y_{1}\right),\left(x_{3}, y_{3}\right)\right)\right) .
\end{aligned}
$$


(6) From Theorem 3.3(13), the pair $\left(I_{3}, I_{6}\right)$ is a pair of $S E$-implications. Moreover, since $\left(x_{1}, y_{1}\right) \leq\left(x_{2}, y_{2}\right)$ iff $y_{1}<y_{2}$ or $y_{1}=y_{2}, x_{1} \leq x_{2}$ iff $I_{3}\left(\left(x_{1}, y_{1}\right),\left(x_{2}, y_{2}\right)\right)=(2,3)$ iff $I_{6}\left(\left(x_{1}, y_{1}\right),\left(x_{2}, y_{2}\right)\right)=(2,3)$, then $T\left(\left(x_{1}, y_{1}\right)\right.$, $\left.\left(x_{2}, y_{2}\right)\right) \leq\left(x_{3}, y_{3}\right)$ iff $\left(x_{1}, y_{1}\right) \leq I_{3}\left(\left(x_{2}, y_{2}\right),\left(x_{3}, y_{3}\right)\right)$ iff $\left(x_{2}, y_{2}\right) \leq I_{6}\left(\left(x_{1}, y_{1}\right)\right.$, $\left.\left(x_{3}, y_{3}\right)\right)$.

(7) From Theorem 3.3(14), the pair $\left(I_{2}, I_{7}\right)$ is a pair of $S E$-implications. Moreover, since $\left(x_{1}, y_{1}\right) \leq\left(x_{2}, y_{2}\right)$ iff $y_{1}<y_{2}$ or $y_{1}=y_{2}, x_{1} \leq x_{2}$ iff $I_{2}\left(\left(x_{1}, y_{1}\right),\left(x_{2}, y_{2}\right)\right)=(2,3)$ iff $I_{7}\left(\left(x_{1}, y_{1}\right),\left(x_{2}, y_{2}\right)\right)=(2,3)$, then $T\left(\left(x_{1}, y_{1}\right)\right.$, $\left.\left(x_{2}, y_{2}\right)\right) \leq\left(x_{3}, y_{3}\right)$ iff $\left(x_{1}, y_{1}\right) \leq I_{7}\left(\left(x_{2}, y_{2}\right),\left(x_{3}, y_{3}\right)\right)$ iff $\left(x_{2}, y_{2}\right) \leq I_{2}\left(\left(x_{1}, y_{1}\right)\right.$, $\left.\left(x_{3}, y_{3}\right)\right)$.

\section{References}

[1] A. Dvurecenskij, Pseudo MV-algebras are intervals in l-groups, J. Australian Math. Soc., 72 (2002), 427-445, doi: 10.1017/s1446788700036806.

[2] A. Dvurecenskij, On pseudo MV-algebras, Soft Computing,5 (2001), 347354, doi: 10.1017/s005000100136.

[3] P. Flonder, G. Georgescu, A. lorgulescu, Pseudo t-norms and pseudo-BL algebras, Soft Computing, 5(2001), 355-371. doi: 10.1017/s005000100136.

[4] N. Galatos, C. Tsinakis, Generalized MV-alebras, J. of Algebra, 283 (2005), 254-291, doi: 10.1016/j. jalgebra,2004,07,002.

[5] G. Georgescu, A. lorgulescu, Pseudo MV-algebras, Multiple-Valued Logics, 6 (2001), 193-215.

[6] G. Georgescu, A. Popescu, Non-commutative fuzzy Galois connections, Soft Computing, 7 (2003), 458-467, doi: 10.1017/s00500-003-0280-4.

[7] G. Georgescu, A. Popescu, Non-commutative fuzzy structures and pairs of weak negations, Fuzzy Sets and Systems, 143 (2004), 129-155, doi: $10.1016 /$ j. fss, $2003,06,004$.

[8] G. Georgescu, A. Popescue, Non-dual fuzzy connections, Arch. Math. Log., 43(2004), 1009-1039, doi: 10.1007/s00153-004-0240-4.

[9] U. Höhle, E. P. Klement, Non-classical logic and their applications to fuzzy subsets , Kluwer Academic Publisher, Boston, 1995, doi: 10.1007/978-94011-0215-5. 
[10] Y.C. Kim, Pairs of interval negations and interval implications, Int. J. Pure and Applied Math. 88(2) (2013), 305-319, doi: 10.12732/ijpam.v88i2.12. 
